\title{
An Investigation on the GRB Peak Energy and Low-Energy Spectral Slope
}

\author{
Hoi-Fung Yu* and Felix Ryde and Hüsne Dereli \\ Department of Physics, Royal Institute of Technology, Sweden \\ The Oskar Klein Centre for Cosmoparticle Physics, Sweden \\ E-mail: dy@kth.se
}

There is no conclusive model for the emission mechanism for Gamma-Ray Bursts (GRBs). The conventional synchrotron emission models have been shown to present difficulties in explaining the hard low-energy spectral slopes [3] and the sharp peak curvature [6] in the observed time-resolved spectra. On the contrary, thermal emission from the photosphere (whether or not re-processed by sub-photospheric dissipation, which results in a broadened Planck function) provides a natural explanation for most of the narrow time-resolved spectra [5, 4]. The peak energy and low-energy spectral slope of some GRB spectra are shown to exhibit correlation [1]. We investigate such behaviour by performing time-resolved spectral analysis using Bayesian techniques [2] and compare the results to various emission models including the thermal emission models.

7th Fermi Symposium 2017

15-20 October 2017

Garmisch-Partenkirchen, Germany

${ }^{*}$ Speaker. 


\section{References}

[1] J. M. Burgess, F. Ryde, H.-F. Yu, Taking the band function too far: a tale of two $\alpha$ 's, MNRAS $\mathbf{4 5 1 . 2}$ (2015) 1511 [arXiv:1410.7647]

[2] G. Vianello, R. J. Lauer, P. Younk, et al., The Multi-Mission Maximum Likelihood framework (3ML), (2015) [arXiv: 1507.08343$]$

[3] R. D. Preece, M. S. Briggs, R. S. Mallozzi, et al., The Synchrotron Shock Model Confronts a "Line of Death" in the BATSE Gamma-Ray Burst Data, ApJ 506.1 (1998) L23 [astro-ph/9808184]

[4] F. Ryde, A. Pe'er, T. Nymark, et al., Observational evidence of dissipative photospheres in gamma-ray bursts, MNRAS 415.4 (2011) 3693 [arXiv: 1103 . 0708]

[5] F. Ryde, The Cooling Behavior of Thermal Pulses in Gamma-Ray Bursts, ApJ 614.2 (2004) 827 [astro-ph/0406674]

[6] H.-F. Yu, H. J. van Eerten, J. Greiner, et al., The sharpness of gamma-ray burst prompt emission spectra, A\&A $\mathbf{5 8 3}$ (2015) A129 [arXiv: 1507.05589 ] 